\title{
Resource-Efficient Production Planning through Flexibility Measurements in Value Creation Systems
}

\author{
Sven Rogalski*, Hendro Wicaksono, and Konstantin Krahtov \\ Institute for Information Management in Engineering, Karlsruhe Institute of Technology, \\ Karlsruhe, Germany \\ \{sven.rogalski, hendro.wicaksono, konstantin.krahtov\}@kit.edu
}

\begin{abstract}
For years, manufacturing companies have faced an increasingly complex and rapidly changing market environment which is the result of, if nothing else, higher customer individualization. This particularly concerns SME's, whose competitiveness is increasingly dependent on the early identification of new customer and market requirements and their ability to dynamically respond to these in an adequate fashion. In order to meet the high standards demanded of the planning quality and planning safety with ever increasing complexity and the continuous reduction of the planning time available, SMEcompatible IT technologies are needed for the simulation of complex manufacturing relations. The following article addresses this problem and in-troduces the method set ecoFLEX, which enables the simulation of targeted and dynamic alignment of existing plant structures, resources and value-added processes with new production requirements. Unlike digital factory planning tools, a complete picture of the specific plant situation is not required, as will be illustrated by selected case studies from the medium sized production sector.
\end{abstract}

Keywords: Flexibility Measurement, Manufacturing Systems, Resource Efficiency, Production Planning and Control.

\section{Introduction}

The transition of modern industrial nations from a post-industrial to an information orientated society has gained considerable momentum in recent years. Global integration due to the explosive spread of modern communication technologies, especially with the advent of the Internet in the early 90s, resulted in an unprecedented level of transparency and integration of national and international markets. This led on the one hand to the development of new markets, while on the other hand, pushed competitors from low-wage countries into the established markets of developed countries, whose influence in their own markets is slowly disappearing [1]. This places new demands on the flexibility and adaptability of companies to successfully master the everchanging, complex strategic and operational requirements in this turbulent market environment [2] [3].

\footnotetext{
* Corresponding author.
} 
One approach to dealing with the increased requirements of this environment is the product- and resource-based networking of companies in the form of individual valuecreation systems, attuned to manufacturing targets. Their goal is to balance the high uncertainty in planning, induced by the turbulent operational field, on an intercompany level through the appropriate configuration of the network. However, the information acquisition and processing of value-creation planning is already very time consuming and complicated, even at a corporate level [4] [5].

In this climate it is apparent that a company's information management is gradually becoming a key competitive advantage and its importance will undoubtedly continue to increase significantly. While the CIO was once purely responsible for the technical provision of data, it now holds the most responsibility for an enterprise's innovation. The effective and efficient management of information is thus largely codecisive in the quick and effective performance of innovation processes and thus crucial for economic success [6] [7].

Despite the rapid increase in importance and value of information, the IT landscapes of many companies are still stuck in the past. While large companies struggle to align their systems and processes with the new conditions, small and medium-sized enterprises often lack integrated systems. This applies particularly to the production, which must actively contribute to securing long-term corporate success. The use of IT takes over the tasks of planning, design, monitoring and controlling of information throughout the product manufacturing. However, there is a lack of effective and efficient information management, especially for SME, which collects and evaluates information from existing ERP, CRM, SCM or digital factory planning systems, in order to integrate the flexibility and adaptability requirements for its own value creation while taking into account the customer and supplier processes [2] [5].

To master the complexity of the competing requirements of the demanded flexibility and adaptability along with the cost, time and quality goals in the product manufacturing, new information technology tools are needed. These must ensure the integration of existing IT solutions in the production environment of companies in order to allow the systematic and mutually manageable information processing for both strategic planning and operations. Such tools can be grouped under the generic term "Digital Factory" and allow the planning, simulation and optimization of products, processes and their associated use of energy, material, personnel and equipment [5] [8]. However, companies make different demands on the degree of detail of the simulations, the expense for the construction of simulation models, the evaluate options and depth of integration with other systems to avoid redundant work. It is particularly difficult for companies to incorporate the system and product life cycle aspects into their economic goals within their product, process and resource planning, to find the best possible degree of flexibility and resource efficiency for its own value creation [9] [4] [5].

\section{State of the Art}

Leading digital factory planning and simulation tools offer an extensive range of functions for simulation, which can calculate complex and especially dynamic business processes in order to make mathematically safe business decisions. They are also 
suitable for the geometric and functional planning of factory layouts, whose production processes can also be planned and optimized in 2D and 3D. This allows for the visualization, simulation and analysis of the technical elements of production with their respective space requirements, but also physically available production area, the prescribed height and weight loads as well as the material and transportation flows in conjunction with the required manufacturing manpower [5] [10] [11]. Significant obstacles are in particular the high acquisition costs for the usage of such systems which threaten a positive cost-benefit ratio. Also their high training costs, due to their high complexity mean that they find little acceptance. Smaller factory planning tools are indeed cheaper than the market leader, but generally inadequately cover many specific planning problems. Further simulations are therefore mainly performed manually and are supported by self-developed software extensions or often by MS Excel. Especially critical here is the dependence of the planning results on the subjective factors involved in planning which often only restrictedly survey the impact of decisions and their consequences. Thus, there are significant weaknesses in the design, dimensioning and personnel allocation of production facilities, based on economic uncertainty and the current overcapacity [5].

In the relevant literature there are various approaches that are isolated from the typical digital factory planning tools, through which attempts are made to evaluate flexibility and adaptability of production [5]. The disadvantage of these approaches and assessment procedures, however, is their lack of prevalence in industry as they relate to restricted areas of concern and do not allow cross-analysis. Reasons for this are on the one hand, the unsolved problem of a generally applicable measurement and evaluation of the flexibility and adaptability.

\section{Flexibility Measurements with ecoFLEX}

Against this background, the flexibility assessment toolbox ecoFLEX was developed. This, thanks to its generically built interface, allows for easy integration with existing IT systems for strategic and operational production planning. This enables extensive information gathering and data processing, through the integrated evaluation methods and analysis algorithms for various planning and control tasks (Figure 1). Via the provided functionality for flexibility analysis, existing weaknesses in the design, dimensioning and staff assignment of production assessed in the context of economic uncertainty and potential capacity fluctuations can be evaluated, so that an economic balance between the prevailing planning uncertainties and the required degree of flexibility in production can be assured. Quantifiable parameters can be calculated with ecoFLEX by using existing resource information and their cost-, time and application dependencies, as for example from ERP-systems. Beyond the state of the art and research, this enables objective flexibility analysis on different levels of value creation systems (Network, factory, workplace, etc.).

Due to the fact that calculation parameters, which can be determined for different types of production systems, form the basis of the flexibility evaluation methodology, it can be applied in different sectors. Its customization to different evaluation 
challenges requires little effort and thus contributes most to the user acceptance of SME. The reason for this is the production network model, which allows the presentation of evaluation-relevant and existing production objects in an abstracted way and that ensures the logical, level-related gathering and structuring of the needed flexibility parameters. This enables the identification of flexibility-related dependencies between single production objects, so that flexibility deficits can be allocated to the responsible unit. The conceptual set-up is graphically represented in the figure 1.

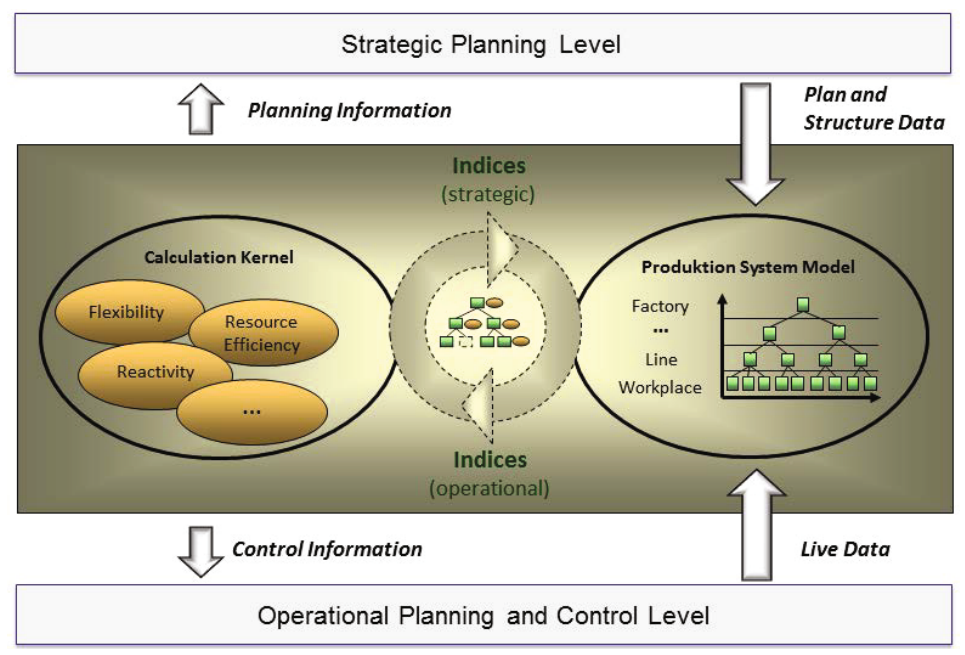

Fig. 1. Overview of the ecoFLEX approach (according to [2])

\section{Application Experience with ecoFLEX}

The usefulness of the Flexibility Assessment Toolbox ecoFLEX has already been proven many times in practice. The basis for this was the previously presented implementation of the evaluation mechanisms as a software tool. An example of the associated benefits and advantages will be shown using the example of a mediumsized automotive supplier for assembly technology. There, two assembly lines, together with workplaces have been investigated, where a total of nine different products for roof mounting are manufactured for the automotive industry. One of these products is available in two variants.

Due to the remarkable scope of analysis and the emerging findings, only the findings obtained with ecoFLEX in Assembly Line 1 will be discussed in this paper. Through the analysis with ecoFLEX, the production volume that allows the production to run economically was determined for the first time. This corresponded to the production numbers of at least 1,286 pieces (break-even amount) and a maximum of 5,596 pieces (maximum production), based on a work week and a defined product mix ratio (see Figure 2, top left). 
All deviations above or below this volume range will inevitably lead to a loss of revenue. Such a break-even analysis was not possible before, because despite a directed material flow, this was discontinuous, which meant that there was a buffer in the form of crates and rolling racks at each work station. In addition, the personnel were not necessarily tied to a specific workplace, but could change between different ones (but not all). This allowed for buffers upstream the product stage to be depleted and replenished to a certain degree. In addition, there were also scattered cycles in the material flow that occurred whenever intermediate products did not meet the prescribed quality standards and were therefore sent back to workplaces upstream for rework. This resulted in a very high complexity and consequently a lack of transparency in production-related dependencies in the production planning of the company, so that resource planning was dependent on a so-called "gut feeling".

This "gut feeling" was also of great importance in the strategic planning of resources. Similarly so for the consideration of the conversion of Assembly Line 1, where the company's production planning found "suspected" flexibility deficits and thus wanted to improve the corresponding sub-optimal work processes. The focus was on the manual riveting workplace of the line (see figure 2, workplace 0060), which could be occupied by up to two operators and which processed the intermediate products from the Assembly Line. According to planning, this workplace should be replaced with an automated workplace (a riveting robot), where the necessary investment costs would be $€ 100,000$. The analysis of this proposed line reconstruction was done by calculating the so-called ecoFLEX index. This index, which represents a flexibility index for a particular factory object, gives information about the manufacturing flexibility in a value-creation system in a simple form. It is not very useful when considered in isolation, but only when compared with the ecoFLEX indices of other factory objects in the scenarios to be examined. Thus, with larger deviations in the indices in the form of "outliers", existing flexibility discrepancies in a value creation system can be detected very quickly and the corresponding factory objects can be classified.

This confirms the aforementioned example of the proposed procurement of a riveting robot to replace the manual workplace 0060. This led, as shown in the flexibility investigation, to a significant increase in the ecoFLEX index from $79.95 \%$ to $94.38 \%$, due to a significant increase in productivity, resulting from reduced scrap and reduced process times. Although this looked very promising at first glance, such a measure would have led to a local flexibility surplus which could not be totally exhausted by the assembly line. Worse still, from the perspective of the assembly line, this measure would have resulted in flexibility losses as a result of high investment costs for the conversion of the line, which would've increased the break-even volume to 1,441 units per week, whereas the maximum production rate could only be able to be increased to 48 units per week. These new findings led to the procurement of the planned riveting robot being discarded. Unexpectedly for the production planner, the real flexibility bottleneck in line 1 was at the workplace 0065, which emerged from the ecoFLEX index calculated (see Figure 2, left side). 


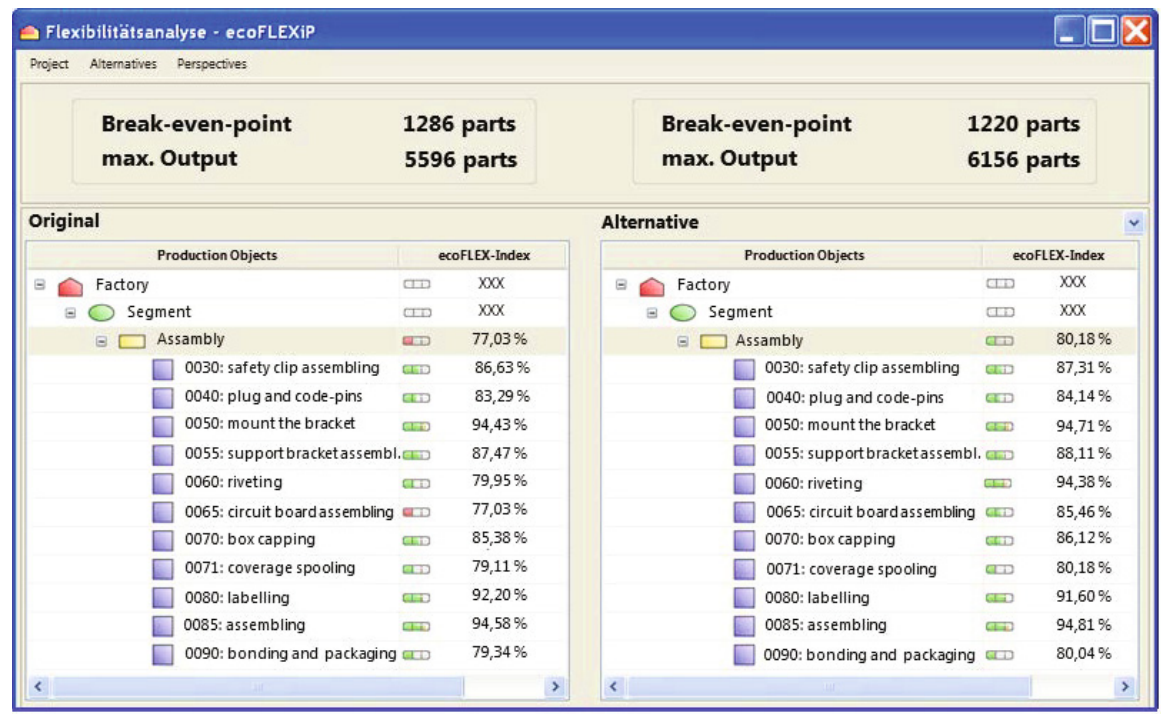

Fig. 2. Analysis of manufacturing flexibility with ecoFLEX

It was found that the operations carried out at this workplace did not have to be exclusively done by specially trained workers, but instead there was the possibility to decouple work operations to allow for different tasks to be done by semi-skilled personnel. This resulted in the skilled work force being freed up for other tasks. To achieve this, only the existing workplace 0065 had to be extended by an additional work bench and buffer storage. Through this an increase in the ecoFLEX index from $77.03 \%$ to $80.18 \%$ resulted for the entire line 1 , which brought a clear improvement of manufacturing flexibility for the line. Expressed in terms of production numbers, this means a reduced break-even quantity of 1,220 pieces, as well as an increased maximum production of 6,156 pieces per workweek (see Figure 2, top right).

As part of these studies it was also found that the dimensioning of the workplaces 0050 and 0085 were chosen as too big in the former planning phase. This is illustrated by the ecoFLEX indices of over 94\%, which as op-posed to workplace 0065 deviate greatly from the average of all ecoFLEX indices (see Figure 2, left panel). The result is a flexibility surplus, which causes avoidable additional costs through the acquisition of both workplaces. This finding was surprising for the production management, which made it clear that it can sometimes be useful to check the so-called "gut feeling" with the appropriate IT tools.

Another consideration made in this context with the ecoFLEX analysis was the assessment of potential outsourcing in order to be able to meet, if necessary, short-term demand spikes that exceed the maximum possible production rate of 6,156 pieces per week. The production management was clear in this case that the work done at workplace 0065 could be outsourced to a production supplier, but lacked a clear overview of the associated chances and risks for Assembly Line 1. In this way, in-house, operations-related production costs of $6.31 €$ occur for the carrying out of special assembly process at workplace 0065 (see Figure 3, right panel). The outsourcing of 
the process to an external third party would mean an increase in the company operations-related costs to $7.35 €$ (see Figure 3, right panel), which are based on the production service costs and logistics costs. Due to the increased variable production costs within the Assembly Line 1, the break-even volume increased to 1,573 pieces per work week, but thereby also increasing the maximum production volume to 7,920 pieces per week.

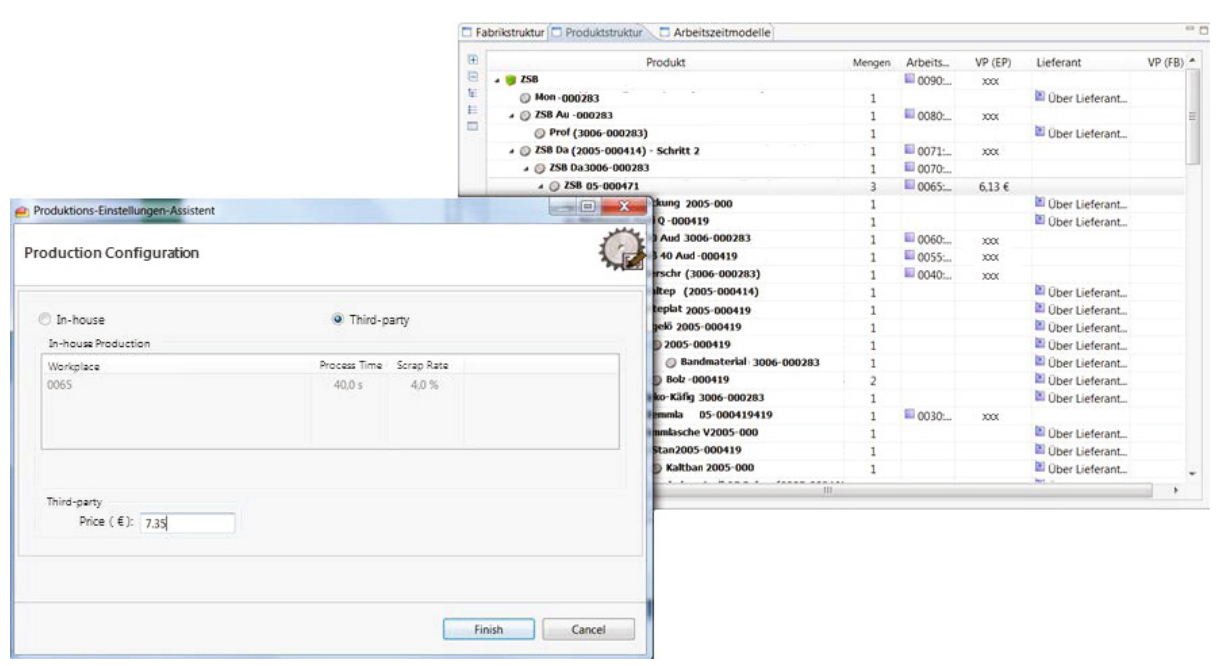

Fig. 3. Outsourcing Analysis with ecoFLEX

The ecoFLEX investigations provided important insights for the production planning of the company, as it was now clear that workplace 0065 represented a flexibility bottleneck that could be solved as described above by the easily accessible process improvement measures, significantly increasing the total flexibility and output rates (6156 units per week) of the assembly line (see Figure 2, top right). To meet sudden demand levels that exceed the existing capacity of the line, there is the possibility of using a production service provider, creating an additional flexibility buffer of 1,764 pieces per week, which can be retrieved when needed with a lead time of approximately three weeks.

\section{Summary}

The ecoFLEX investigations provided important insights for the production planning of the company, as it was now clear that workplace 0065 represented a flexibility bottleneck that could be solved as described above by the easily accessible process improvement measures, significantly increasing the total flexibility and output rates $(6,156$ units per week) of the assembly line (see Figure 2, top right). To meet sudden demand levels that exceed the existing capacity of the line, there is the possibility of using a production service provider, creating an additional flexibility buffer of 1,764 
pieces per week, which can be retrieved when needed with a lead time of approximately three weeks.

On this basis, cost-driven flexibility and capacity weaknesses in the complete plant structure are quickly identified and solved through both operational and strategic measures. Taking into account the total efficiency of the production, strategic production changes can be quickly simulated by a simple integration of existing expertise of the company's own production planning and control. Thus, unnecessary additional costs due to inefficiencies in resource usage or senseless adjustments to the production infrastructure, such as construction and reconstruction of production facilities are avoided, creating financial flexibility for future investments.

A recent coupling of ecoFLEX with a 3D Visualisation and simulation solution allowed an immersive virtual reality of the production facilities. Production planners now have the opportunity to examine in detail the inefficiencies in resource allocation identified with ecoFLEX in the virtual plant models and to develop alternative solutions that would otherwise only be possible using a real object. With the help of the virtual image of the production and the simulation of processes, it is possible to test different strategies of dealing with their impact on the entire system.

\section{References}

1. Ehrlenspiel, K.: Integrierte Produktentwicklung: Denkabläufe, Methodeneinsatz, Zusammenarbeit. Karl HanserVerlag, München (2003)

2. Rogalski, S., Wicaksono, H.: Handling Resource Efficiency in Production of Small and Medium sized Enterprises. In: 18th International Conference on Engineering, Technology and Innovation (ICE 2012), Munich, Germany, June 18-20 (2012)

3. Loeffler, C., Westkämper, E., Unger, K.: Änderungsdynamik und Varianz im Automobilbau Analyse der Produktvarianz und deren Auswirkung auf die Produktion. Wtwerkstattstechnik Online 3, 99-104 (2011)

4. Reinhart, G., von Bredow, M.: Bewertung von Kunden-Lieferanten-Beziehungen in der Automobilindustrie. ZWF Zeitschrift für Wirtschaftlichen Fabrikbetrieb 12, 832-836 (2008)

5. Rogalski, S.: Factory design and process optimisation with flexibility measurements in industrial production. International Journal of Production Research, 1--12 (2012)

6. Maass, W.: Elektronische Wissensmärkte: Handel von Information und Wissen über digitale Netze. GablerVerlag (2009)

7. Schwinn, K.: Informationsmanagementprozesse im Unternehmen. In: Daten-und Informations-qualität, pp. 260-276 (2011)

8. Bracht, U., Geckler, D., Wenzel, S.: Digitale Fabrik Methoden und Beispiele. Springer, Heidelberg (2011)

9. Kunst, S.: Wirtschaftlichkeit der Virtual Reality Technologie. VdmVerlag Dr. Müller, Saarbrücken (2007)

10. Kohler, U.: Methodik zur kontinuierlichen und kostenorientierten Planung produktionstechnischer Systeme. Herbert Utz Verlag, München (2007)

11. Westkämper, E.: Digital Manufacturing in the Global ERA. Digital Enterprise Technology, Part 1, 3-14 (2007) 